\title{
Photonic crystal nanocavity laser in an optically very thick slab
}

\author{
Se-Heon Kim, ${ }^{1,2, *}$ Jingqing Huang, ${ }^{1,2}$ and Axel Scherer ${ }^{1,2}$ \\ ${ }^{1}$ Department of Electrical Engineering, California Institute of Technology, Pasadena, California 91125, USA \\ ${ }^{2}$ Kavli Nanoscience Institute, California Institute of Technology, Pasadena, California 91125, USA \\ *Corresponding author: seheon@caltech.edu
}

Received November 15, 2011; accepted December 9, 2011;

posted December 15, 2011 (Doc. ID 158271); published February 6, 2012

\begin{abstract}
A photonic crystal (PhC) nanocavity formed in an optically very thick slab can support reasonably high- $Q$ modes for lasing. Experimentally, we demonstrate room-temperature pulsed lasing operation from the PhC dipole mode emitting at $1324 \mathrm{~nm}$, which is fabricated in an InGaAsP slab with thickness $(T)$ of $606 \mathrm{~nm}$. Numerical simulation reveals that when $T \geq 800 \mathrm{~nm}$, over $90 \%$ of the laser output power couples to the PhC slab modes, suggesting a new route toward an efficient in-plane laser for photonic integrated circuits. (c) 2012 Optical Society of America

OCIS codes: $230.5298,250.5960$.
\end{abstract}

An optically thin dielectric slab with photonic crystal $(\mathrm{PhC})$ air holes has been a versatile platform for designing various high- $Q$ cavities [1]. Thickness $(T)$ of the $\mathrm{PhC}$ slab is often chosen to maximize the size of the photonic band gap (PBG) [2], which is approximately equal to half the effective wavelength of the cavity resonance. For designing a PhC slab laser emitting at $1.3 \mu \mathrm{m}$, this thickness consideration requires that $T$ should be about $250 \mathrm{~nm}$.

In this Letter, we show that even a very thick slab can support sufficiently high- $Q$ cavity modes for lasing. Once we are free from the thickness constraint, design of a current-injection type laser becomes more feasible; we can employ a vertically varying $p$-i-n structure along with a current confinement aperture, as has been done for vertical-cavity surface-emitting lasers [3]. Furthermore, as will be shown below, we can build an efficient in-plane emitting laser, where most of the laser emission couples to the two-dimensional (2D) Bloch modes [2] in the $\mathrm{PhC}$ slab.

We begin with numerical simulations using the finitedifference time-domain (FDTD) method. We adopt the widely used modified single-cell cavity design [4] and investigate the $\mathrm{PhC}$ dipole mode as shown in Fig. 1 . We assume $T$ and the lattice constant $(a)$ are $200 \overline{0}$ and $305 \mathrm{~nm}$, respectively. The refractive index of the slab is assumed to be 3.4. Other structural parameters are as follows [4]: the background hole radius $(R)=0.35 a$, the modified hole radius $(R m)=0.25 a$, and the hole radius perturbation $(R p)=0.05 a$. It should be noted that the in-plane PBG [2] is completely closed at $T \approx 1.5 a$ for a PhC slab with $R=0.35 a$. However, it is interesting that we can still find several resonant modes that seem to be well confined within the defect region, as shown in Figs. 1(b),(c). In fact, these modes have the same transverse mode profile, while the number of intensity lobes along the $z$ direction varies from one to three. Therefore, these modes originate from the slab resonance between the top and bottom surfaces, which can act as reflectors due to the relatively high refractive index of the slab. We summarize various optical characteristics of the dipole modes in a slab with $T=2,000 \mathrm{~nm}$, including $Q$, emission wavelength $\lambda$, and mode volume $V$, in Table $\underline{1}$ [ $\underline{4}$ ]. In particular, $Q_{\text {tot }}[5]$ of the fundamental mode is over 5000 . It should be noted that a similar thick slab design was proposed by Tandaechanurat et al. with a special focus on a PhC cavity in a $T=1.4 a$ slab [] ].

To gain further insight into the loss mechanism, in Fig. 2, we calculate $Q_{\text {tot }}, Q_{\text {vert }}$, and $Q_{\text {horz }}[1,5]$ as a function of $T$, where $a$ is varied to keep the emission wavelength at $1.3 \mu \mathrm{m}$. First, let us focus on $Q_{\text {vert }}$. In the case of a thin slab PhC cavity, $Q_{\text {vert }}$ depends strongly on $R m$ and $R p$ [7] and the $Q_{\text {vert }}$ of the dipole mode can be as high as $\sim 15,000$ [4]. Indeed, when $T \leq 400 \mathrm{~nm}, Q_{\text {vert }}$ is in the range of 10,000 . However, when $T \geq 500 \mathrm{~nm}, Q_{\text {vert }}$ increases almost exponentially as $T$ increases. We obtain a surprisingly high $Q_{\text {vert }}$ of $6 \times 10^{5}$ at $T=2,000 \mathrm{~nm}$, implying the existence of a certain highly efficient vertical confinement mechanism, which will be clarified later. On the other hand, the in-plane confinement mechanism is not very effective, as expected, because the $\mathrm{PBG}$ is closed for $T>\sim 450 \mathrm{~nm}$. However, $Q_{\text {horz }}$ can be brought up to $\sim 5500$ at $T=2,000 \mathrm{~nm}$, and $Q_{\text {tot }}$ is usually limited by $Q_{\text {horz }}$ at large $T$. This large difference between $Q_{\text {vert }}$
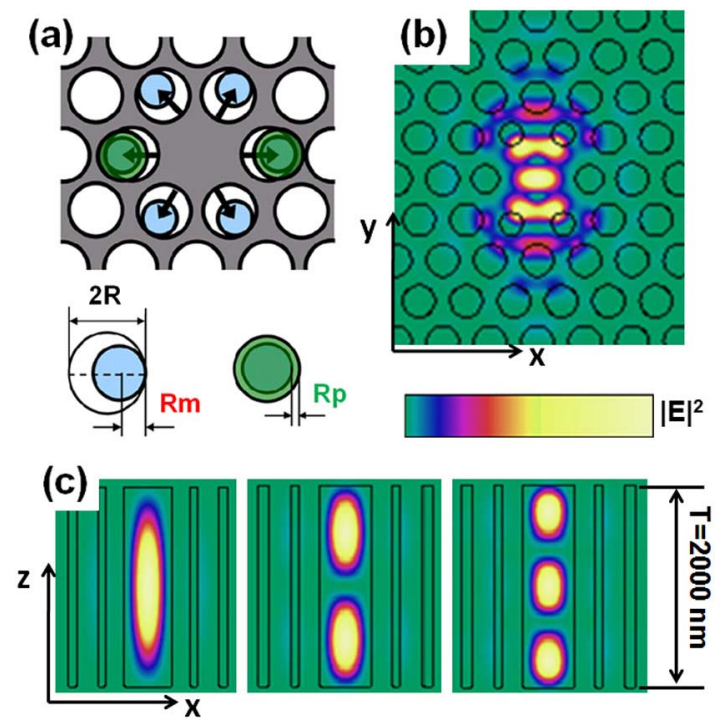

Fig. 1. (Color online) (a) Design of the modified dipole cavity, (b), (c) FDTD simulations for the dipole mode in a PhC slab with $T=2,000 \mathrm{~nm}$ : (b) top-down view of the electric-field intensity $\left(|\mathbf{E}|^{2}\right)$ profile and (c) cross-sectional views of $|\mathbf{E}|^{2}$ of the fundamental, first-order, and second-order slab modes. 
Table 1. Optical Properties of the Higher-Order Slab Modes

\begin{tabular}{lcrcc}
\hline & $\lambda(\mathrm{nm})$ & $Q_{\text {tot }}$ & $Q_{\text {vert }}$ & $V(\lambda / n)^{3}$ \\
\hline Fundamental & 1324 & 5392 & $6.5 \times 10^{5}$ & 2.45 \\
First order & 1305 & 1582 & 41,600 & 2.65 \\
Second order & 1275 & 755 & 27,900 & 2.86 \\
\hline
\end{tabular}

and $Q_{\text {horz }}$ implies that most of the photons generated inside the cavity will leak into the $\mathrm{PhC}$ slab; at $T=2,000 \mathrm{~nm}$, over 99\% (horizontal emission efficiency, $\left.\eta_{\text {horz }}=1-Q_{\text {tot }} / Q_{\text {vert }}\right)$ of the total number of photons will be funneled through the PhC slab. $\eta_{\text {horz }}$ is over $90 \%$ when $T \geq 800 \mathrm{~nm}$. This behavior is completely opposite to the case of a thin slab cavity, where $Q_{\text {horz }}$ can increase indefinitely by simply adding more layers of $\mathrm{PhC}$ barriers; therefore, $Q_{\text {tot }}$ is limited by $Q_{\text {vert }}$.

To better understand the highly effective vertical confinement mechanism, let us now consider a hypothetical PhC slab cavity with $T=\infty$. The resulting structure may be viewed as a $\mathrm{PhC}$ fiber [8], and thus one can define a waveguide dispersion in the $z$ direction. In Fig. 3(a), we show the waveguide dispersion of the dipole mode. It should be noted that these modes are not PBG-guided except for the $k_{z}=0$ point, because a nonzero wave vector $\left(k_{z}>0\right)$ breaks the TE/TM symmetry, and the original 2D PhC structure with $R=0.35 a$ cannot have a complete PBG for both TE and TM [8]. Thus, the guided modes with $k_{z}>0$ are inherently lossy. Now we will show that the observed three resonant modes in Fig. 1(c) originate from these guided modes. In Fig. 3(a), we show intersection points between the dispersion curve and the three normalized frequencies $\left(\omega_{n}=a / \lambda\right)$ of the resonant modes. We find that these points are almost equally arranged in the $k$ space, where $\Delta k_{z}$ indeed satisfies the Fabry-Perot resonance condition, $\Delta k_{z}=\pi / T ; \Delta k_{z} /$ $(2 \pi / a)=a /(2 T) \approx 0.076$ [9]. Note that the group velocity $\left(V_{g} \equiv d \omega / d k\right)$ of the fundamental dipole mode will approach zero as $T \rightarrow \infty$ and $k_{z} \rightarrow 0$ [See Fig. 3(b)].

In view of this waveguide model, the $Q_{\text {tot }}$ of the fundamental dipole mode can be written as the sum of waveguide propagation loss and scattering loss at the two mirror facets such that $[\underline{9}, \underline{10}]$

$$
\frac{1}{Q_{\mathrm{tot}}}=\frac{V_{g}}{\omega}\left[\alpha+\frac{1}{T} \log \left(\frac{1}{r_{0}^{2}}\right)\right] .
$$
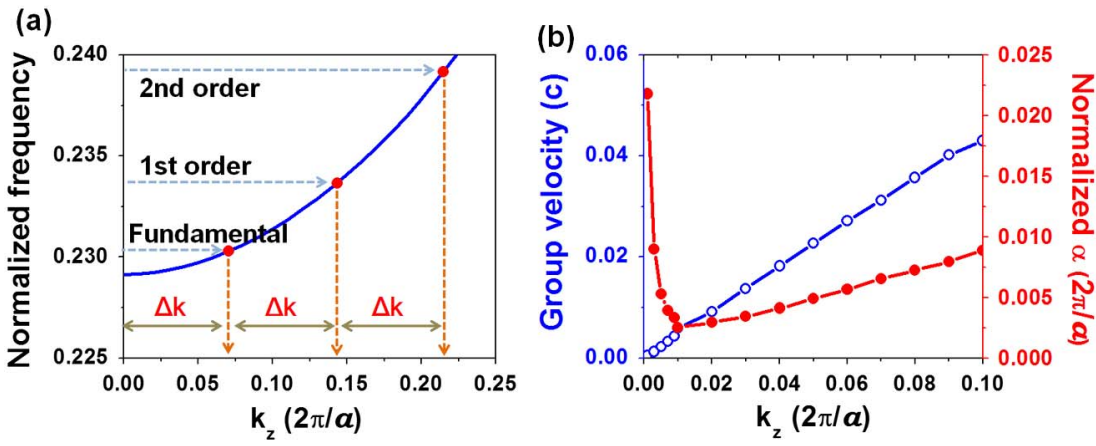

Fig. 3. (Color online) (a) Waveguide dispersion along the $z$ direction for the dipole mode. The normalized frequencies of the three dipole resonant modes shown in Fig. 1(c) are overlaid on the dispersion curve. (b) Group velocity $\left(V_{g}\right)$ and waveguide propagation loss coefficient, $\alpha$, simulated by FDTD. $V_{g}$ and $\alpha$ are normalized by $c$ (speed of light) and $2 \pi / a$, respectively. 

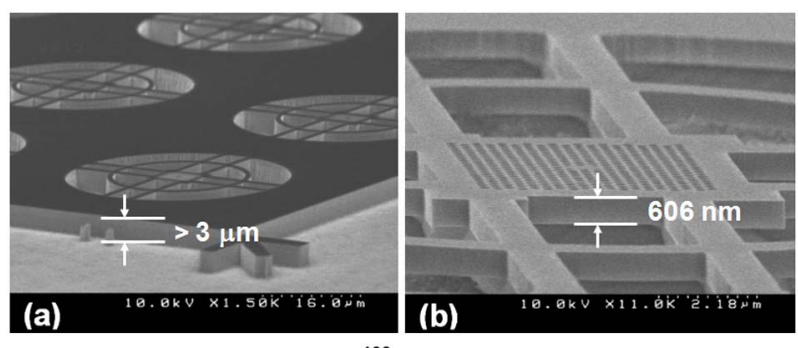

(c)
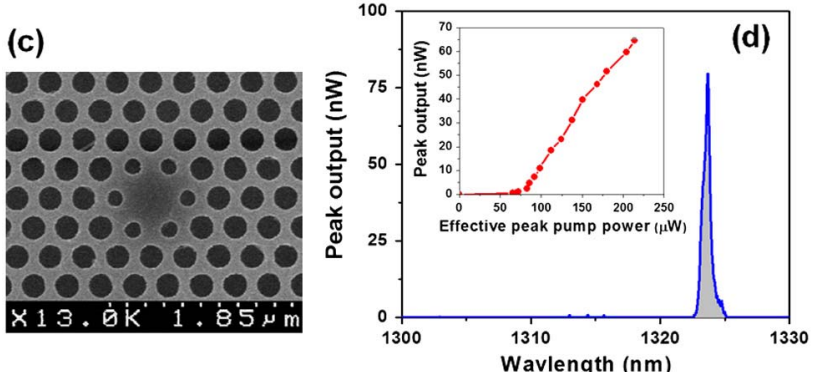

Fig. 4. (Color online) (a)-(c) SEM images of PhC dipole lasers formed in a $606 \mathrm{~nm}$ InGaAsP slab. (a) Our dry-etching capability enables very deep (>3 $\mu \mathrm{m})$ and vertical etching; (b) a tilted image taken after selective wet-chemical etching; (c) a top view of the fabricated laser device. (d) Characteristics of the laser device.

of the slab, with $120 \AA$ thick tensile-strained (-0.3\%) $1.12 \mu \mathrm{m}$ InGaAsP barriers in between. $240 \mathrm{~nm}$ thick unstrained $1.12 \mu \mathrm{m}$ InGaAsP is on the top and bottom of the active layer and serves as a cladding. We use standard nanofabrication processes including electron-beam lithography (using hydrogen silsesquioxane as the resist), dry etching to drill the $\mathrm{PhC}$ air holes, and selective wetchemical etching to undercut the InP sacrificial layer. To define deep and vertical air holes, we use hightemperature $\left(190{ }^{\circ} \mathrm{C}\right) \mathrm{Ar} / \mathrm{Cl}_{2}$ chemically assisted ionbeam etching (CAIBE). As shown in Figs. 4(a) and (b), our optimized CAIBE system produces very deep $(>3 \mu \mathrm{m})$ and vertical sidewalls, which are requisites to experimentally realize a theoretical $Q_{\text {tot }}$ of $2000-3000$. Figs. 4(b) and (c) show scanning electron microscope (SEM) images of fabricated laser devices.

The fabricated lasers are photopumped at room temperature with an $830 \mathrm{~nm}$ laser diode. The repetition rate of the pump laser is $1 \mathrm{MHz}$ with a duty cycle of $2 \%$. We use a $100 \times$ objective lens to focus the pump laser on to the cavity region. The same objective lens is used to collect the emitted laser light, which is fed into an optical

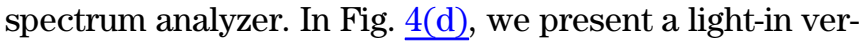
sus light-out $(L-L)$ curve and a lasing spectrum for one example laser device. We confirm that the laser emission indeed comes from one of the degeneracy-split dipole modes [1] by comparing the emission wavelength $(1323.7 \mathrm{~nm})$ with that obtained by FDTD simulation.
Assuming that about $20 \%$ of the actual incident pump power is absorbed in the slab, the effective threshold peak pump power is estimated to be $78 \mu \mathrm{W}$.

Though the present work merely demonstrates an optically pumped device, it is our hope that the thick slab $\mathrm{PhC}$ cavity design will provide versatile routes toward a current-injection $\mathrm{PhC}$ laser. One feasible plan is to place the whole PhC slab cavity onto a metal substrate, where the metal may serve as both an electrical current pathway and a heat sink [14]. An alternative is to take advantage of the increased slab thickness, which enables more flexible design of the p-i-n doped layers and a current aperture structure.

The authors would like to acknowledge support from the Defense Advanced Research Projects Agency under the Nanoscale Architecture for Coherent Hyperoptical Sources program under grant \#W911NF-07-1-0277 and from the National Science Foundation through NSF CIAN ERC under grant \#EEC-0812072.

\section{References and Notes}

1. O. Painter, J. Vučkovič, and A. Scherer, J. Opt. Soc. Am. B 16, 275 (1999).

2. S. G. Johnson, S. Fan, P. R. Villeneuve, J. D. Joannopoulos, and L. A. Kolodziejski, Phys. Rev. B 60, 5751 (1999).

3. K. Iga, Jpn. J. Appl. Phys. 47, 1 (2008).

4. S.-H. Kim, S.-K. Kim, and Y.-H. Lee, Phys. Rev. B 73, 235117 (2006).

5. $Q_{\text {tot }}$ is defined by the decay rate of the total electromagnetic energy stored in the cavity such that $U(t)=$ $U(0) \exp \left[-\omega t / Q_{\mathrm{tot}}\right]$. Then, the total radiation power $\left(\sim 1 / Q_{\text {tot }}\right)$ can be decomposed into power radiated into the PhC slab $\left(\sim 1 / Q_{\text {horz }}\right)$ and power radiated in the out-of plane direction $\left(\sim 1 / Q_{\mathrm{vert}}\right)$; therefore, $1 / Q_{\mathrm{tot}}=1 / Q_{\mathrm{horz}}+1 / Q_{\mathrm{vert}}$.

6. A. Tandaechanurat, S. Iwamoto, M. Nomura, N. Kumagai, and Y. Arakawa, Opt. Express 16, 448 (2008).

7. H.-Y. Ryu, M. Notomi, and Y.-H. Lee, Appl. Phys. Lett. 83, 4294 (2003).

8. J. D. Joannopoulos, S. G. Johnson, J. N. Winn, and R. D. Meade, Photonic Crystals: Molding the Flow of Light, 2nd ed. (Princeton University, 2008).

9. S.-H. Kim, G.-H. Kim, S.-K. Kim, H.-G. Park, Y.-H. Lee, and S.-B. Kim, J. Appl. Phys. 95, 411 (2004).

10. L. A. Coldren and S. W. Corzine, Diode Lasers and Photonic Integrated Circuits (Wiley, 1995).

11. Y. Tanaka, T. Asano, Y. Akahane, B.-S. Song, and S. Noda, Appl. Phys. Lett. 82, 1661 (2003).

12. E. Kuramochi, M. Notomi, S. Hughes, A. Shinya, T. Watanabe, and L. Ramunno, Phys. Rev. B 72, 161318 (2005).

13. M. Ibanescu, S. G. Johnson, D. Roundy, Y. Fink, and J. D. Joannopoulos, Opt. Lett. 30, 552 (2005).

14. S.-H. Kim, J. Huang, and A. Scherer, "From vertical-cavities to hybrid metal/photonic-crystal nanocavities: towards high-efficiency nanolasers," http://arxiv.org/abs/1109.0103 (2011). 